\title{
Performance analysis of regularization algorithms used for image reconstruction in computed tomography
}

\author{
D. WYSOCZAŃSKI*, J. MROCZKA, and A.G. POLAK \\ Chair of Electronic and Photonic Metrology, Faculty of Electronics, Wroclaw University of Technology, \\ 53/55 Prusa St., 50-317 Wrocław, Poland
}

\begin{abstract}
Computed tomography is one of the most significant diagnostic techniques in medicine. This work is focused on hard-field imaging, where signals take a form of straight rays and the reconstructed image can be presented as a matrix with unknown pixels. Algebraic methods for direct computation of the image have not been used in practice because of the scale of the problem and numerical errors appearing in the solution. The aim of this work was to analyse the performance of direct algebraic algorithms for tomographic image reconstruction including regularisation mechanism such as: generalised regularisation, Tikhonov regularisation, Twomey regularisation and ridge regression (RR), as well as comparing the results with the filtered backprojection (FBP) as the reference method. The performed analyses demonstrated that the regularised algebraic methods are more accurate than the commonly used FBP, and RR appeared the most precise among them. Additionally it was shown that the invariant system matrix (inverted during calculations) can be easily determined by solving the forward problem. Finally, potential directions of further research have been pointed out.
\end{abstract}

Key words: computed tomography, image reconstruction, algebraic approach, regularization methods.

\section{Introduction}

Computed tomography (CT) is one of the most significant and simultaneously still most dynamically developing diagnostic techniques in medicine nowadays, thus refining the image reconstruction methods is of a great practical importance. Tomography can be differentiated into hard-field and soft-field types. In hard-field tomography (as X-ray, magnetic resonance or emission one) it is assumed that a high-energy signal propagates rectilinearly and its attenuation is primary caused by local energy absorption [1]. Conversely, in soft-field tomography (as electrical impedance or optical diffusion one), also other phenomena play a crucial role and the signals propagate simultaneously between a source and a detector on many ways dependent on local properties on an object under investigation [2]. Knowing the distribution of these properties as well as the nature of the excitation used, it is possible to apply the ray-tracing technique for finding the trajectory of a signal beam between the transmitter and the receiver (as in microwave or ultrasound tomography) [3].

This work focuses on hard-field tomography, where the signal takes a form of a straight ray. In such conditions, carrying out the discretisation of an investigated section, it is possible to unambiguously associate the ray between the transmitter and the receiver with definite image pixels (and determining its width - also with a coincident fraction of a given pixel area). In effect, the analysed section can be associated with a matrix (the system matrix) of coefficients that are related to measured data by linear system of algebraic equations [1]. In practice (if possible) overdetermined systems of equations are used, i.e. with the number of measurements ex- ceeding the number of unknowns. Algebraic methods for direct computation of the image (involving the inversion of the system matrix), though conceptually straightforward, have not been used because of the scale of the solved problem (large matrices), resulting in hardware (RAM resources) and time limits, as well as in numerical ill-conditioning, significantly reducing the signal-to-noise ratio (SNR) in the reconstructed image. However, a lot of iterative methods have been elaborated. The most important group of such algorithms is based on the Kaczmarz projection [4], developed further by Tanaba [5]. The advantage of the iterative calculations relies on effective solving the large systems of equations, and the shortcoming is an approximate solution reached [1]. The most popular variants of the iterative algorithms include: the algebraic reconstruction technique (ART) [6], simultaneous iterative reconstructive technique (SIRT) [7], or simultaneous algebraic reconstruction technique (SART) [3]. Another group of algorithms for iterative solving the systems of equations utilise the Gauss-Siedel method [8-10]. Their advantage follows from the possibility of taking into account additional prior informant on the reconstructed image during succeeding iterations.

The use of prior information enables the reduction of distortions in the retrieved image. In the algorithms of tomographic image reconstructions, most frequently it takes the form of: nonnegativity of pixels [8,10-12], a penalty term for the non-smoothness of the solution [9-11, 13-17], or a reference image $[18,19]$. This prior knowledge plays a particular role in statistical iterative reconstruction (SIR) algorithms, used for emission (PET and SPECT) and low-dose $\mathrm{X}$-ray tomography, where the image is retrieved from an undetermined system of equations, most often by applying knowl-

*e-mail: dariusz.wysoczanski@pwr.wroc.pl 
edge on stochastic character of data and the Bayesian approach $[9,14,17,20,21]$. The regularization mechanism is also used to eliminate distortions following a strong correlation of data recorded by receiving coils in parallel magnetic resonance imaging (MRI) [19, 22].

Dynamic development of computing technology, including the use of multicore graphics processing units (GPU) and comprehensive random access memories, prompts nowadays to re-interest in direct algebraic methods. Moreover, the algorithms for matrix inversion enable to include the regularization mechanisms and to reduce the level of disturbances in retrieved images. The quality of a reconstructed image depends, however, not only on the regularization method itself, but also on the regularization parameter. Too small its value leaves visible distortions in the image, and too large blurs edges and details. Recently a method for automated selection of the regularization parameter value, minimizing the total mean-square error of reconstruction, was proposed [23].

The aim of this work is to analyse the performance of the algorithms for tomographic image reconstruction by direct inversion of the system matrix, including the regularisation methods as: generalised, Tikhonov, Twomey regularisations and ridge regression, as well as comparing the results with filtered backprojection as the reference method. The aforementioned method for automated selection of the regularization parameter was applied. The paper shows also that in hard-field tomography the system matrix is invariant (depending only on the image size) and that the forward model can be simulated to determine its elements instead of analyzing tediously the system geometry. Moreover, this matrix can be calculated only once and used many times for a given tomograph, fastening computations.

At the beginning of the article (Sec. 2), the theoretical fundamentals of the forward and inverse mapping in hard-field tomography (including the discrete approach) are presented, together with the regularization methods for image reconstruction, the used method for the selection of optimal regularisation parameter, the fast method for system matrix computation and the performed simulation experiments. Section 3 contains the results achieved, and their analysis is presented in Sec. 4. In the end, the main conclusions following from the study are presented.

\section{Methods}

2.1. The forward and inverse algorithms. In hard-field tomography, where one can assume rectilinear propagation of a physical signal between the source and the detector, the relation of measured ray attenuation on a way $s$ to absorbing properties $f$ of the object specified at each point of its section $(x, y)$, can be described by integral operator. The emitted (in parallel or fan geometry) beam of rays allows recording one projection $P(z, \varphi)$ of object's properties on the $Z$ axis, forming an angle $\varphi$ with the $X$ axis of the Cartesian coordinate system. This mapping is described by continuous Radon's transform [1]:

$$
\begin{gathered}
P(z, \phi)=\int_{\operatorname{ray}(z, \phi)} f(x, y) d s \\
=\int_{a_{y}}^{b_{y}} \int_{a_{x}}^{b_{x}} f(x, y) \delta(x \cos \phi+y \sin \phi-z) d x d y,
\end{gathered}
$$

where $\delta$ is Dirac's delta, and $\left[a_{x}, b_{x}\right]$ and $\left[a_{y}, b_{y}\right]$ are the coordinates limiting the image area in the $X Y$ system. The Radon transform can be recognized as a continuous forward model for hard-field tomography, and the projection values derived for succeeding angles (analytically or numerically), known as a sinogram, as a solution of the forward problem.

The inverse Radon transform is also known. It shows how to find the distribution $f(x, y)$ from measured projections $P(z, \varphi)$. It is not however applied in practice, since the required derivatives $\partial P / \partial z$ must be calculated from disturbed data, that leads to large errors in the retrieved image. In practice, the filtered backprojection method (FBP) is used [1]. It relies on the calculation of the Fourier transform of succeeding projections $P(z, \varphi)$ and thereafter filtering them by a filter developed in the frequency domain. In effect, a set of filtered Fourier transforms is achieved. The transforms are folded radially at subsequent angles $\varphi$, so the encoded by them image possesses well defined slowly-variable properties (background) and poorly defined fast-variable features (edges). Thus, before the final image reconstruction, a chosen method of interpolation is used to calculate evenly distributed values of the 2D FFT transform. The FBP, giving the inverse problem solution, is a standard method for tomographic image reconstruction, and it has been chosen as the reference method in this study.

Besides the continuous Radon transform, also a discrete approach for describing the projections can be applied. This approach requires the image area to be subdivided into $n \times n$ pixels with averaged absorptions $f_{j}(j=1,2, \ldots, N$, where $N=n^{2}$ ) and to assume a narrow, but finite width of the ray (it can be related to a detector size for instance). Then the projection $p_{i}$ for the $i$-th ray $(i=1,2, \ldots, M)$ is given by:

$$
p_{i}=\sum_{j=1}^{N} w_{i j} f_{j},
$$

where the weight $w_{i j}$ is proportional to the joint section of the $i$-th ray and the $j$-th pixel area [1]. Thus, the total discrete forward model for rectilinear tomography has the following matrix form:

$$
\mathbf{p}=\mathbf{W} \mathbf{f},
$$

where $\mathbf{p}=\left[p_{i}\right]_{M \times 1}, \mathbf{W}=\left[w_{i j}\right]_{M \times N}$ and $\mathbf{f}=\left[f_{j}\right]_{N \times 1}$.

The derived algebraic approach to tomography indicates an alternative way of the inverse problem solving - the image reconstruction by matrix inversion. To reduce noise in the retrieved image, an overdetermined number of equations is usually used $(M>N)$, so the pseudoinverse matrix should be employed to find pixel values $\widehat{\mathbf{f}}[2]$ :

$$
\widehat{\mathbf{f}}=\left(\mathbf{W}^{T} \mathbf{W}\right)^{-1} \mathbf{W}^{T} \mathbf{p},
$$


an operation equivalent to minimising the functional $V(\mathbf{f})=$ $\|\mathbf{p}-\mathbf{W f}\|_{2}^{2}$.

The discrete approach, besides it is a straightforward concept, is also easy for computer implementation. Unluckily, the required matrix inversion is numerically ill-conditioned in tomography, that leads to uncontrolled propagation of augmented disturbances form recorded projections $\mathbf{p}$ into the reconstructed image $\widehat{\mathbf{f}}$. Reduction of this effect can be achieved by regularisation of the inverse problem.

2.2. Regularization algorithms. There are plenty of approaches yielding the decrease of disturbances appearing in the inverse problem solution when the matrix inversion is numerically ill-conditioned. They were overviewed e.g. in $[24,25]$. One of them imposes constraints on the searched solution by completing the functional $V$ by the penalty term $\Omega$. This leads to the following form of regularised reconstruction procedure:

$$
\begin{gathered}
\widehat{\mathbf{f}}_{R}=\arg \min _{\mathbf{f}} V_{R}(\mathbf{f}), \\
V_{R}(\mathbf{f})=V(\mathbf{f})+\gamma \Omega(\mathbf{f}),
\end{gathered}
$$

where $\gamma$ is the regularisation parameter. The general form of the penalty term is as follows:

$$
\Omega(\mathbf{f})=\left\|\mathbf{D}\left(\mathbf{f}-\mathbf{f}_{*}\right)\right\|_{2}^{2},
$$

where $\mathbf{D}$ is a discrete operator called the regularisation matrix, and $\mathbf{f}_{*}$ is a reference image. Recently it has been shown that popular regularisation methods are specific cases of the above described inverse problem [23], and they are analysed in this study.

Applying the Tikhonov method $\left(\mathbf{f}_{*}=\mathbf{0}\right)[26,27]$, matrix D was chosen as a spatial differential operator of the 1 st order (minimising the difference between a given pixel and its four neighbours with conjoint edges):

$$
\widehat{\mathbf{f}}_{T H}=\left(\mathbf{W}^{T} \mathbf{W}+\gamma \mathbf{D}^{T} \mathbf{D}\right)^{-1} \mathbf{W}^{T} \mathbf{p} .
$$

In the Twomey method [28] $(\mathbf{D}=\mathbf{I})$, the image retrieved by fast FBP was taken as $\mathbf{f}_{*}$ (minimisation of the difference between the reconstructed image and $\mathbf{f}_{*}$ ):

$$
\widehat{\mathbf{f}}_{T W}=\left(\mathbf{W}^{T} \mathbf{W}+\gamma \mathbf{I}\right)^{-1}\left(\mathbf{W}^{T} \mathbf{p}+\gamma \mathbf{f}_{*}\right) .
$$

A next method, the ridge regression [29] ( $\mathbf{D}=\mathbf{I}$ and $\left.\mathbf{f}_{*}=\mathbf{0}\right)$, minimises the amplitude of the reconstructed image:

$$
\widehat{\mathbf{f}}_{R R}=\left(\mathbf{W}^{T} \mathbf{W}+\gamma \mathbf{I}\right)^{-1} \mathbf{W}^{T} \mathbf{p} \text {. }
$$

The last of the analysed approaches was the generalised regularisation [23], taking into account both the regularization matrix $\mathbf{D}$ and the reference image $\mathbf{f}_{*}$ :

$$
\widehat{\mathbf{f}}_{G R}=\left(\mathbf{W}^{T} \mathbf{W}+\gamma \mathbf{D}^{T} \mathbf{D}\right)^{-1}\left(\mathbf{W}^{T} \mathbf{p}+\gamma \mathbf{D}^{T} \mathbf{D} \mathbf{f}_{*}\right) .
$$

The algebraic methods make the use of the system matrix $\mathbf{W}$, which is determined by the awkward geometrical analysis of interactions between the ray and pixels. This can be avoided noticing that: 1) in rectilinear tomography $\mathbf{W}$ is identical to the sensitivity matrix $\mathbf{X}$ of the forward model (3):

$$
\mathbf{X}=\frac{\partial \mathbf{p}}{\partial \mathbf{f}}=\mathbf{W}
$$

and 2) $\mathbf{X}$ does not depend on the reconstructed image (since the model is linear) but its size, and it can be determined once and used many times in a given tomograph. Exploiting the aforementioned properties, matrix $\mathbf{X}$ can be easily computed numerically (without geometrical analyses), employing the forward model $\mathbf{p}(\mathbf{f})$ and any image $\mathbf{f}_{0}$ of an appropriate size:

$$
\begin{gathered}
\mathbf{x}_{j}=\frac{\mathbf{p}\left(\mathbf{f}_{0}+\Delta \mathbf{f}\right)-\mathbf{p}\left(\mathbf{f}_{0}\right)}{\Delta f_{j}} \\
\mathbf{X}=\left[\begin{array}{llll}
\mathbf{x}_{1} & \mathbf{x}_{2} & \ldots & \mathbf{x}_{N}
\end{array}\right]
\end{gathered}
$$

where $\Delta \mathbf{f}$ is a vector consisting merely of zeros, except the $j$-th element equal to $\Delta f_{j}$, since then, according to (3):

$$
\begin{aligned}
& \mathbf{x}_{j}=\frac{\mathbf{W}\left(\mathbf{f}_{0}+\Delta \mathbf{f}\right)-\mathbf{W} \mathbf{f}_{0}}{\Delta f_{j}}=\frac{\mathbf{W} \cdot \Delta \mathbf{f}}{\Delta f_{j}} \\
& =\frac{\mathbf{W} \cdot\left[\mathbf{0} \Delta f_{j} \mathbf{0}\right]^{T}}{\Delta f_{j}}=\frac{\mathbf{w}_{j} \cdot \Delta f_{j}}{\Delta f_{j}}=\mathbf{w}_{j} .
\end{aligned}
$$

A numerical implementation of the Radon transform was utilized to calculate $\mathbf{p}\left(\mathbf{f}_{0}+\Delta \mathbf{f}\right)$ and $\mathbf{p}\left(\mathbf{f}_{0}\right)$.

\subsection{Automatic selection of the regularization parameter.} Many approaches for the selection of regularisation parameter $\gamma$ have been proposed for the last decades. In this work, a recently published method reducing the mean-square error of reconstruction has been applied [23]. This can be done by choosing $\gamma$ that minimises the following functional $V_{\gamma}$ :

$$
V_{\gamma}(\gamma)=\boldsymbol{\varepsilon}^{T}(\gamma) \boldsymbol{\varepsilon}(\gamma)+\widehat{\boldsymbol{\sigma}}^{T}(\gamma) \mathbf{W}^{T} \mathbf{W} \widehat{\boldsymbol{\sigma}}(\gamma),
$$

where $\varepsilon=\mathbf{p}-\mathbf{W} \widehat{\mathbf{f}}$ is a residual vector and $\widehat{\boldsymbol{\sigma}}$ is the standard deviation of the estimator of $\widehat{\mathbf{f}}$ assessed by cross-validation. This approach enables an automated selection of $\gamma$ dependent on the noise level presented in the recorded projections $\mathbf{p}$.

Searching the optimal $\gamma$ was done iteratively for each image reconstruction case. The procedure began with the calculation of $V_{\gamma}$ for $\gamma_{0}=0.01$ and then was repeated with 10times increased or decreased $\gamma$, until the following condition was fulfilled: $V_{\gamma}\left(0.1 \cdot \gamma_{i}\right)>V_{\gamma}\left(\gamma_{i}\right)<V_{\gamma}\left(10 \cdot \gamma_{i}\right)$. In the end, the parabola crossing through these three points was found and the optimal $\gamma_{*}$ at parabola's minimum was calculated.

2.4. Simulation studies. Numerical simulations were performed in Matlab 7.5.0 (R2007b) with the use of chosen procedures from the Image Processing Toolbox: modified Shepp-Logan phantom, Radon transform, filtered backprojection (FBP) and pseudorandom generator of Gaussian noise. The aim of these investigations was to determine the influence of the noise level on the error of image reconstruction by selected methods: FBP, generalized regularization (GR), Tikhonov regularization (RTH), Twomey regularization (RTW) and ridge regression (RR).

During simulations the test image in the form of the Shepp-Logan phantom [30] was used after its normalization in the range of $[0,1]$. The typical resolution of tomographic images amounts to $512 \times 512$ or $256 \times 256$ pixels for on-line 
reconstructions. The latest high-resolution arrays possesses up to $768 \times 768$ and $1024 \times 1024$ pixels. Taking into account, however, the time of repeated Monte Carlo simulations, the phantom resolution was set to $25 \times 25$ pixels during calculations and $50 \times 50$ pixels for imaging. Sinograms were determined using the Radon transform in the angle range of 0-179 degrees with the step of 1 degree.

Each resulting sinogram was corrupted by simulated additive Gaussian noise. From data presented in [31] it follows that the level of noise induced by measurement chain in lowdose tomography does not exceed $6 \%$. Thus, the impact of noise levels of $0.1-10 \%$ was analysed in this study (the noise level was defined as the ratio of noise standard deviation to the maximal value in a sinogram).

Firstly, the quality of an image reconstruction by FBP for different parameters of this procedure was analysed to optimise it for the case under investigation (the image reconstructed by FBP constituted the reference during the evaluation of remaining methods). Diverse interpolation methods were checked: linear, nearest-neighbour, spline and cubic, as well as the original Ram-Lak filter or the Ram-Lak filter modified by sine and cosine functions, or Hamming and Hann windows.

To compare the analysed methods, the measure of reconstruction quality was assumed as the relative reconstruction error $\delta$ :

$$
\delta=\frac{\|\widehat{\mathbf{f}}-\mathbf{f}\|_{2}}{\|\mathbf{f}\|_{2}} \cdot 100 \%=\frac{\sqrt{\sum_{i=1}^{N}\left(\widehat{f}_{i}-f_{i}\right)^{2}}}{\sqrt{\sum_{i=1}^{N} f_{i}^{2}}} \cdot 100 \%,
$$

where f denotes the original image (undisturbed phantom) and $\widehat{\mathbf{f}}$ is an image retrieved by a reconstruction method (such defined measure is equivalent to the Frobenius norm for $2 \mathrm{D}$ images).

The 100 image reconstructions were simulated for each of investigated methods from the sinograms disturbed by realisations of noise at succeeding noise levels. Further calculations were done directly with the resulting images as well as with images normalised in the range $[0,1]$. Finally, the statistical

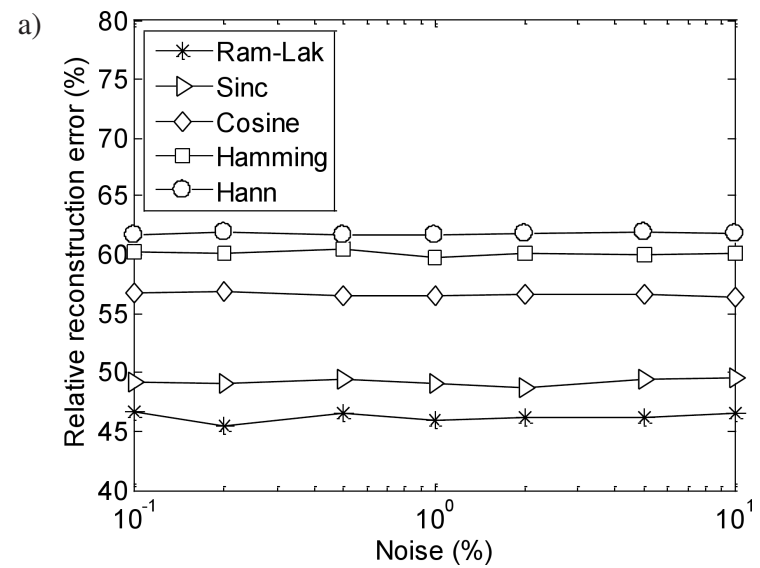

analysis of reconstruction quality was performed computing the mean values and standard deviations of the relative error of reconstruction for each group of 100 results.

\section{Results}

Among diverse options of the FBP algorithm, the original Ram-Lak filter appeared the best choice (Fig. 1). Simultaneously, the most accurate results were received with the spline interpolation (Fig. 1a). However, the time of simulations with spline interpolation was longer than with a slightly less precise linear interpolation by an order, so the comparative image for succeeding studies was produced using FBP with the Ram-Lak filter and linear interpolation.

The results of simulations investigating the influence of noise level on the accuracy of image reconstruction for the analysed methods is shown in Fig. 2 (without normalization of the resulting image) and Fig. 3 (with normalisation in the range of $[0,1])$. The quality of reconstruction achieved with the regularised methods decreases with the increase of a noise level, however in normalised images this effect is visible only over the threshold of about $2 \%$, when using FBP (Fig. 3a). In each case the results are also more scattered at higher noise levels (Figs. 2b and 3b), reaching larger values (about 1\%) for the regularised methods than for FBP, being however less dependent on the noise level in the former instance. Simultaneously, the scatter is smaller between images not normalised. In this study all regularised methods appeared more accurate than FBP for non-normalised images (Fig. 2a), as well as in the case of normalised images for noise levels lesser than about $1.5 \%$ (Fig. 3a). Among them, the ridge regression (RR) was slightly better than the others.

In Fig. 4 the phantom image $(F)$ is presented together with exemplary images reconstructed by FBP (with the Ram-Lak filter and linear interpolation) and the investigated regularised methods: GR, RTH, RTW and RR (with the automatically selected regularisation parameter). The images achieved with the regularised methods are characterised by a larger sharpness than FBP and, despite smaller values of the relative reconstruction errors at the noise level of $0.5 \%$, visible distortions of random character.

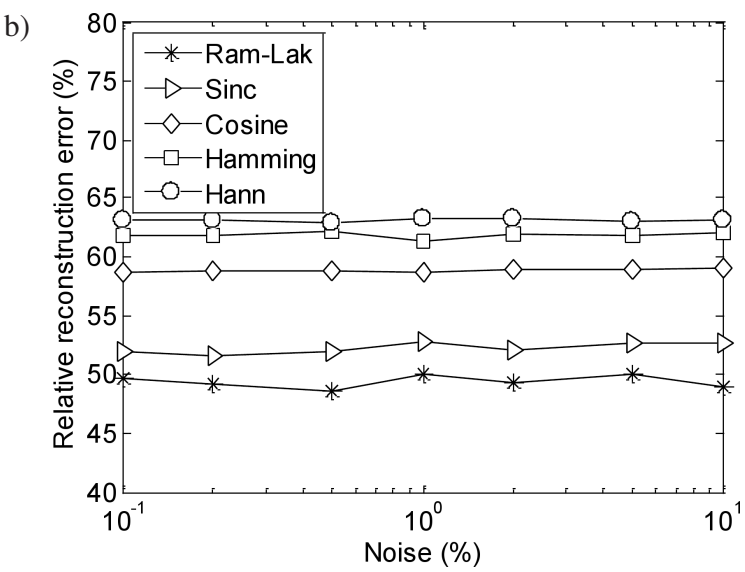

Fig. 1. Dependence of the relative error of image reconstruction on the noise level and the filter type for the filtered backprojection method (FBP) with different interpolations: a) spline, b) linear (see text for details and abbreviations) 
Performance analysis of regularization algorithms used for image reconstruction...

a)

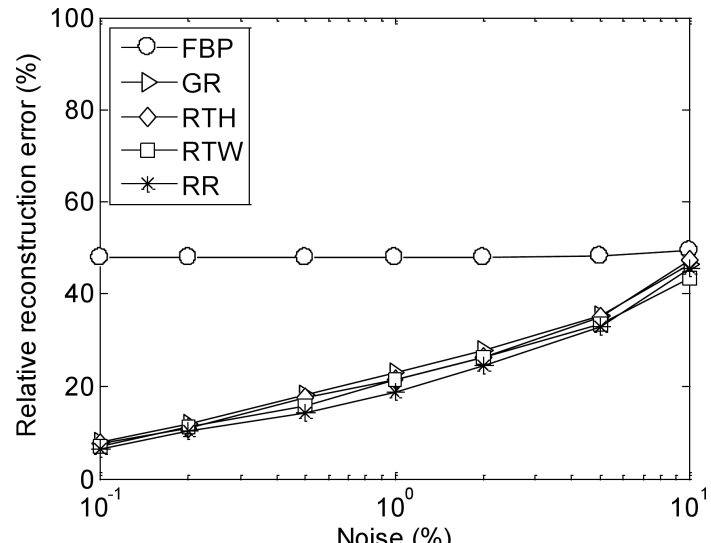

b)

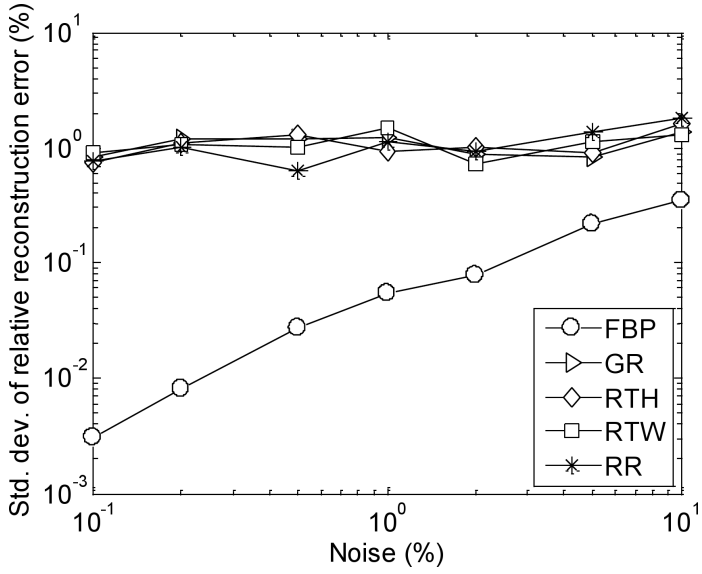

Fig. 2. Impact of noise level on the mean value (a) and standard deviation (b) of the relative error of reconstruction in relation to methods used - the case of non-normalised images (see text for abbreviations)

a)

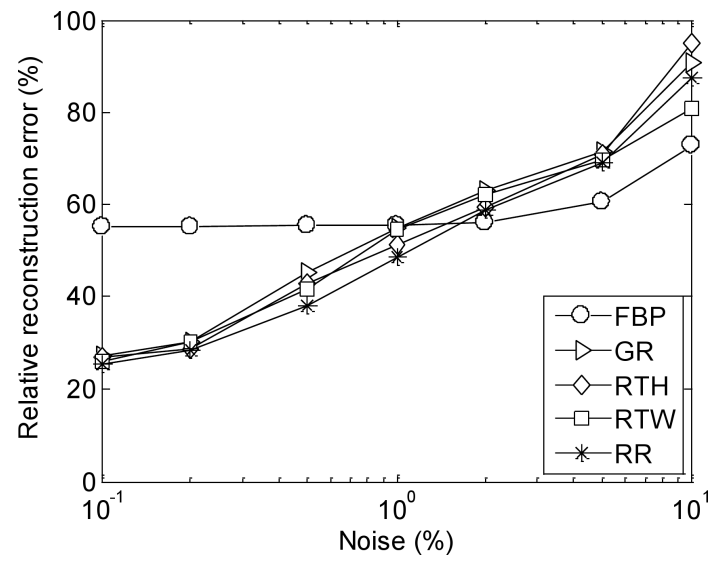

b)

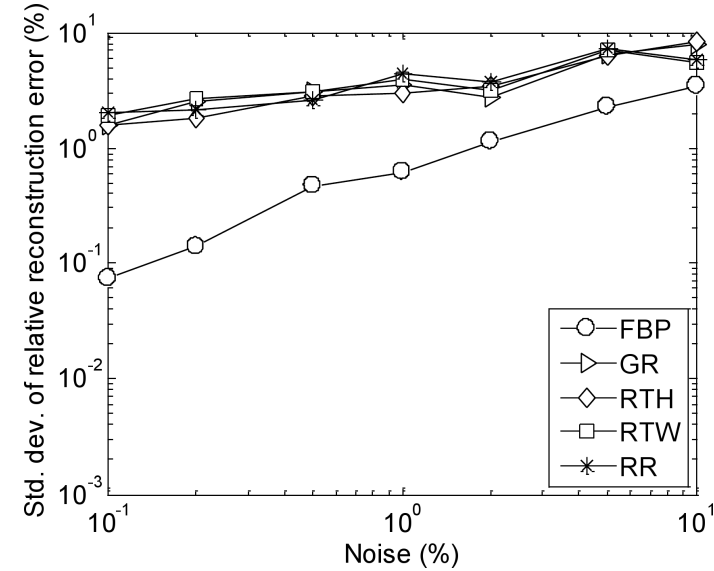

Fig. 3. Impact of noise level on the mean value (a) and standard deviation (b) of the relative error of reconstruction in relation to methods used - the case of normalised images (see text for abbreviations)
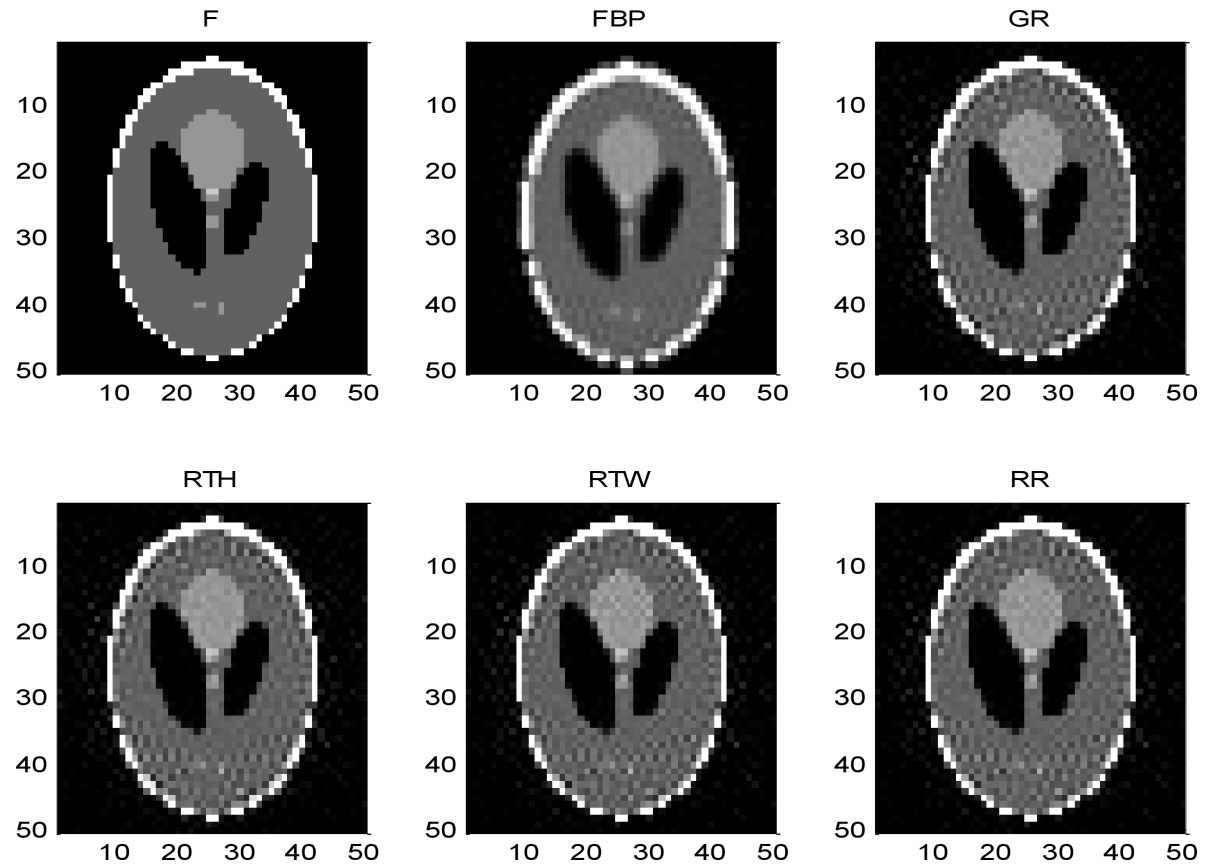

Fig. 4. The original phantom image (F) and the exemplary non-normalised images reconstructed with different methods for the noise level of $0.5 \%$ : filtered backprojection (FBP), generalised regularisation (GR), Tikhonov regularisation (RTH), Twomey regularisation (RTW) and ridge regression $(\mathrm{RR})$ 


\section{Discussion}

The aim of this study was to analyse the accuracy of CT image reconstruction by unused in practice algebraic methods, with a direct inversion of the system matrix and simultaneously including basic regularisation mechanisms. The motivation for this work arose from dynamically growing up computational possibilities of contemporary computers as well as from the progress in the regularisation methods development.

The all-important result following from the simulation investigations is the observation that $\mathrm{CT}$ image reconstruction by regularised algebraic methods is more accurate (in terms of the relative reconstruction error) than the commonly applied filtered backprojection method (FBP) within the whole range of analysed noise levels $(0.1-10 \%)$ in the case of nonnormalised images and for noise levels up to $1.5 \%$ when normalization is applied. This indicates the potential of the methods investigated in this study.

The inclusion of penalty term in the minimised functional, Eq. (5), for the regularisation of the resulting solution is not a new approach. In previous works on tomographic image reconstruction, other authors have used both the Tikhonov [8, $10,15]$ or Twomey [19] regularisation. The innovative element of the approach presented in is paper is the use of regularisation methods together with the direct matrix inversion instead of using it in iterative algorithms.

All of the analysed regularisation methods yielded a similar quality of CT image reconstructions (Figs. 2 and 4). A somewhat surprising outcome is the smallest error (thought the differences are tiny) achieved from ridge regression (RR) (Figs. 2 and 4), since this method does not take advantage of prior information neither in the form of a reference image (as in RTW), nor in the form of local image homogeneity (as in RTH). In contrary, the generalised regularisation method (GR), taking into account both of these mechanisms, belongs to the less accurate methods in this group. The source of this effect consists in a specific nature of the reconstructed image as well as in RR itself. In CT images a lot of pixels have small values, what stems from the presence of background as well as from low-absorbing character of some tissue (dark areas in Fig. 4). The penalty term in RR, Eq. (9), has a form that forces minimisation of pixel values. This particularly enhances the reconstruction quality of images possessing a large number of pixels with small values, as in the phantom used. Analysing the ridge regression one can find that it is equivalent to RTW with the reference image consisting only of zero-valued pixels $\left(\mathbf{f}_{*}=\mathbf{0}\right)$, and to RTH insensitive to the differences between adjacent pixels $(\mathbf{D}=\mathbf{I})$. Simultaneously, the regularisation matrix D used in RTH imposed the minimisation of differences between horizontal and vertical pixels only (as in [10]), however in future studies it can be extended to include also the diagonal neighbours $[8,16]$, that may improve the quality of RTH reconstruction. Taking into account the results achieved for RR and the character of CT images (smooth areas with occasional discontinuities), it is worth also to pay attention to methods smoothing areas with a locally uniform absorption level (representing given tissue), as the total variance method (TV) which takes into account a zero gradient in such regions $[11,12]$. Similarly, for the physical reasons, the inclusion of pixel non-negativity constraint should be also considered [8, 10-12, 32].

Distortions present in the reconstructed image may urge to its normalisation (the range $[0,1]$ was used in this study) before the stage of imaging in a greyscale. The data from Fig. 3 suggest that in such a case, for noise exceeding $1.5 \%$, the FBP method will be more precise than the regularised algebraic methods. This effect is, however, deceptive. Analysing the section of the phantom and the exemplary reconstructed images shown in Fig. 5, it is apparent that normalisation, especially for a higher noise level in the data (bottom panel), reduces the dynamics of reconstructed image, primarily increasing the systematic component of the error (the difference between the reconstructed image and the phantom). Since images reconstructed by RR are less smoothed than the ones yielded by FBP, their dynamics is more suppressed by normalisation, thus the systematic error is increased. This explains the difference between Fig. 2a and Fig. 3a. Additionally, the value of normalising coefficient depends on the difference between the maximal and minimal pixels in the image, which values are random due to the stochastic character of noise. This explains why the scatter of normalised images at a given noise level is bigger than of non-normalised ones (compare Figs. $2 b$ and $3 b$ ).

The major disadvantage of algebraic methods is a long computational time (in simulations performed it was longer from FPB by more than 3 orders) as well as hardware limitations (mainly the size of addressable RAM). In particular, the regularised methods involve a long time of matrix inversion, Eqs. (7-10), as well as of searching the optimal value of regularisation parameter $\gamma$, Eq. (14). Because both the procedures have a crucial impact on reconstruction quality, further work with the aim to shorten the time of computations is needed. The possibility of embedding increasingly bigger RAMs in contemporary computers and operating systems, as well as of organising parallel computations on multicore GPUs [15], may appear helpful to this end.

Another problem is that the random distortions apparent in images reconstructed by regularised algebraic methods can be a bigger diagnostic obstacle than the blurring of edges in FBP, since such disturbances falsely suggest tissue heterogeneity. They can be reduced by increasing the value of $\gamma$, however then (as in FBP) the image becomes more fuzzy [23, 24] (Figs. 4 and 5). Meanwhile, the less sharp edges obstruct the accurate localisation of borders between healthy and diseased tissue. Facilitating the operator with the possibility of user-adjustment of $\gamma$ seems to be an accommodating solution for this problem. 

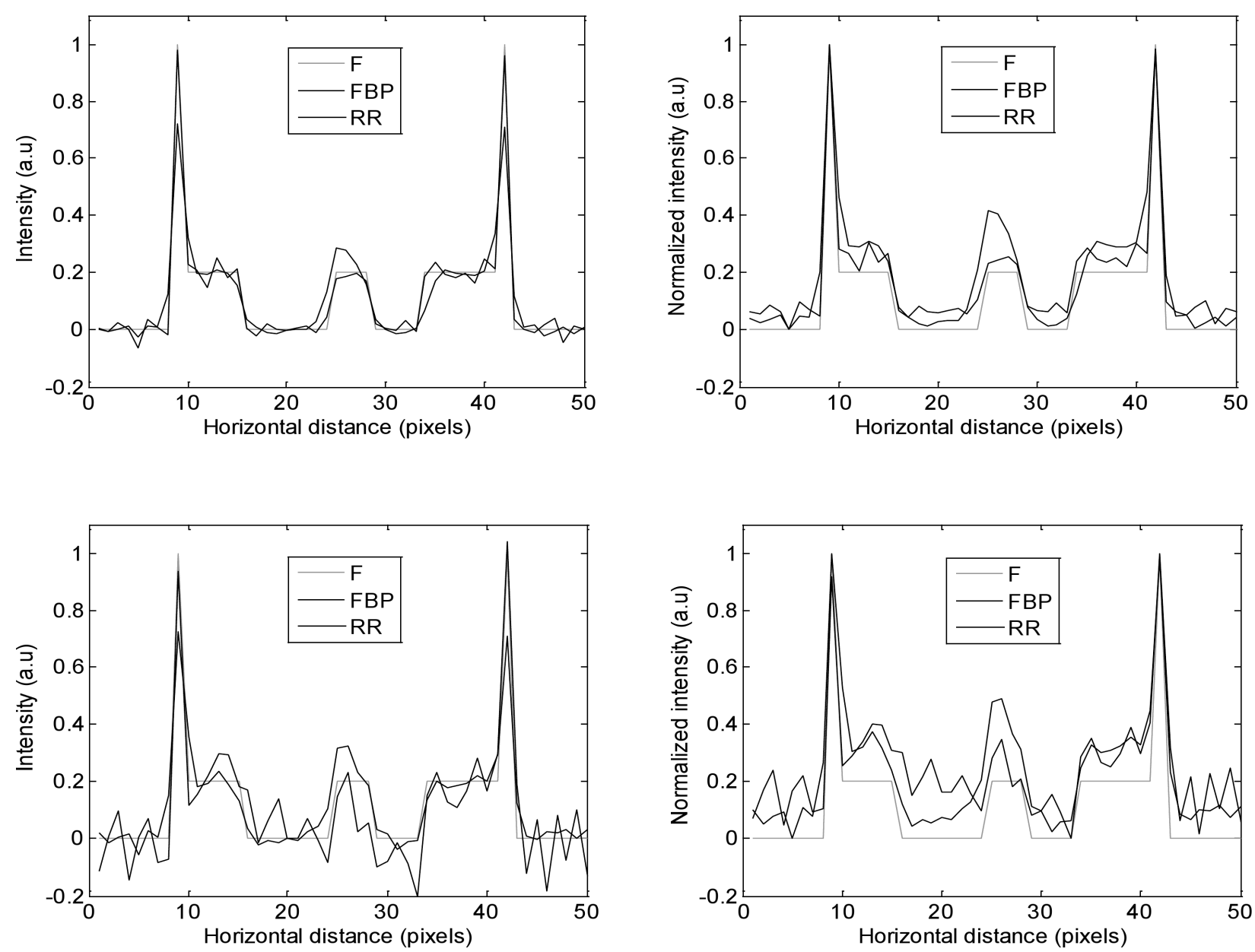

Fig. 5. Central horizontal sections of the phantom $(\mathrm{F})$ and the examples of images reconstructed by filtered backprojection (FBP) and ridge regression (RR) for the noise level of $0.5 \%$ (top panel) and 5\% (bottom panel) without normalisation (left panel) and with image normalisation (right panel)

\section{Conclusion}

The analysed regularised algebraic methods for CT image reconstruction lead to uncomplicated, one-step computational algorithms. In the paper it has been shown that the invariant system matrix (playing an important role in the algorithms) can be easily determined in the case of hard-field tomography by twofold solution of the forward problem. The performed simulations demonstrated also, in spite of time-consuming calculations and a certain level of random distortions in retrieved images, that the regularised algebraic methods are more accurate than the commonly used filtered backprojection one.

The ridge regression method appeared the most precise among the tested regularised algorism, yielding slightly smaller reconstruction errors than the other algebraic approaches. This result followed mainly from the properties of the Shepp-Logan phantom used in investigations. Simultaneously this outcome indicated the significant features of tomographic images, qualifying the type and way of application of prior knowledge to regularise the image reconstruction, as e.g. relatively large and coherent areas with similar absorption (representing given tissue).
The performed preliminary study pointed at the regularised algebraic method, being effective in terms of CT image reconstruction quality. It also entitles to define potential directions of further research that should cover: refining the algorithms in terms of computational effectiveness, modification of regularisation mechanism to include the low-level of image background and presence of coherent homogeneous areas, as well as utilising the fact that the character of noise in a given tomograph can be assessed experimentally and then used to select the optimal value of regularisation parameter.

The achieved results clearly indicate that the analysed methods can play a significant role in improving the quality of CT imaging.

\section{REFERENCES}

[1] A.C. Kak and M. Slaney, Principles of Computerized Tomographic Imaging, IEEE Press, New York, 1988.

[2] D.S. Holder (Ed.), Electrical Impedance Tomography, Institute of Physics Publishing, Bristol, 2005.

[3] A.H. Andersen and A.C. Kak, "Simultaneous algebraic reconstruction technique (SART): a superior implementation of the ART algorithm”, Ultrasonic Imaging 6, 81-94 (1984). 
[4] S. Kaczmarz, "Angenäherte auflösung von systemen linearer gleichungen”, Bull. Intern. Acad. Pol. Sci. Lett. A 35, 355357, (1937).

[5] K. Tanabe, "Projection method for solving a singular system of linear eguations and its applications", Numer. Math. 17, $203-$ 214 (1971).

[6] R. Gordon, R. Bender, and G.T. Herman, "Algebraic reconstruction techniques (ART) for three-dimensional electron microscopy and x-ray photography", J. Theor. Biol. 29, 471-481 (1970).

[7] P. Gilbert, "Iterative methods for the three-dimensional reconstruction of an object from projections", J. Theor. Biol. 36 (1), 105-117 (1972).

[8] J.A. Fessler, "Penalized weighted least-squares image reconstruction for positron emission tomography", IEEE Trans. Med. Imaging 13, 290-300 (1994).

[9] K. Sauer and C. Bouman, "A local update strategy for iterative reconstruction from projections", IEEE Trans. Signal Proc. 41, 534-548 (1993).

[10] P. Sukovic and N.H. Clinthorne, "Penalized weighted leastsquares image reconstruction for dual energy X-ray transmission tomography", IEEE Trans. Med. Imaging 19, 1075-1081 (2000).

[11] E.Y. Sidky, C.M. Kao, and X. Pan, "Accurate image reconstruction from few-views and limited-angle data in divergent-beam CT”, J. X-Ray Sci. Technol. 14, 119-139 (2006).

[12] E.Y. Sidky and X. Pan, "Image reconstruction in circular conebeam computed tomography by constrained, total-variation minimization", Phys. Med. Biol. 53, 4777-4807 (2008).

[13] H.R. Shi and J.A. Fessler, "Quadratic regularization design for 2-D CT", IEEE Trans. Med. Imaging 28 (5), 645-656 (2009).

[14] J.W. Stayman and J.A. Fessler, "Regularization for uniform spatial resolution properties in penalized-likelihood image reconstruction", IEEE Trans. Med. Imaging 19 (6), 601-615 (2000).

[15] S.S. Stone, J.P. Haldar, S.C. Tsao, W.W. Hwu, B.P. Sutton, and Z.P. Liang, "Accelerating Advanced MRI Reconstructions on GPUs.", J. Parallel Distrib. Comput. 68 (10), 1307-131 (2008).

[16] J. Sunnegårdh and P.E. Danielsson, "Regularized iterative weighted filtered backprojection for helical cone-beam CT", Med. Phys. 35 (9), 4173-4185 (2008).

[17] J.B. Thibault, K.D. Sauer, C.A. Bouman, and J. Hsieh, "A three-dimensional statistical approach to improved image quality for multislice helical CT", Med. Phys. 34 (11), 45264544 (2007).
[18] G. Gindi, M. Lee, A. Rangarajan, and I.G. Zubal, "Bayesian reconstruction of functional images using anatomical information as priors", IEEE Trans. Med. Imaging 12 (4), 670-680 (1993).

[19] L. Ying, D. Xu, and Z.P. Liang, "On Tikhonov regularization for image reconstruction in parallel MRI", Conf. Proc. IEEE EMBS 2, 1056-1059 (2004).

[20] S. Holte, P. Schmidlin, A. Lindén, G. Rosenqvist, and L. Eriksson, "Iterative image reconstruction for positron emission tomography: a study of convergence and quantitation problems", IEEE Nucl. Sci. 37, 629-635 (1990).

[21] E.A. Rashed and H. Kudo, "Statistical image reconstruction from limited projection data with intensity priors", Phys. Med. Biol. 57 (7), 2039-2061 (2012).

[22] F.H. Lin, K.K. Kwong, J.W. Belliveau, and L.L. Wald, "Parallel imaging reconstruction using automatic regularization", Magn. Reson. Med. 51 (3), 559-567 (2004).

[23] A.G. Polak, "An error-minimizing approach to regularization in indirect measurements", IEEE Trans. Instrum. Meas. 59 (2), 379-386 (2010).

[24] J. Mroczka and D. Szczuczyński, "Inverse problems formulated in terms of first-kind Fredholm integral equations in indirect measurements", Metrol. Meas. Syst. 16 (3), 333-357 (2009).

[25] J. Mroczka and D. Szczuczyński, "Simulation research on improved regularized solution of the inverse problem in spectral extinction measurements", Applied Optics 51 (11), 1715-1723 (2012).

[26] D.L. Phillips, "A technique for the numerical solution of certain integral equations of the first kind", J. ACM 9, 84-97 (1962).

[27] A.N. Tikhonov, "Solution of incorrectly formulated problems and the regularization method", Sov. Math. Dokl. 4, 1035-1038 (1963).

[28] S. Twomey, "On the numerical solution of Fredholm integral equations of the first kind by the inversion of the linear system produced by quadrature", J. ACM 10, 97-101 (1963).

[29] A.E. Hoerl, "Application of ridge analysis to regression problems", Chem. Eng. Progress 58, 54-59 (1962).

[30] L.A. Shepp and B.F. Logan, "The Fourier reconstruction of a head section", IEEE Trans. Nucl. Sci. 21, 21-43 (1974).

[31] C. Jiao, D. Wang, and H. Lu, "Multiscale noise reduction on low-dose CT sinogram by stationary wavelet transform", IEEE Nucl. Sci. Symp. Conf. 1, 5339-5344 (2008).

[32] J. Mroczka and D. Szczuczyński, "Improved regularized solution of the inverse problem in turbidimetric measurements", Applied Optics 49 (24), 4591-4603 (2010). 